\title{
Misoprostol for miscarriage management in a woman with previous five cesarean deliveries: a case report and literature review
}

This article was published in the following Dove Press journal:

Therapeutics and Clinical Risk Management

9 May 2017

Number of times this article has been viewed

\author{
Doua AISaad ${ }^{1,2}$ \\ Sawsan Alobaidly ${ }^{3}$ \\ Palli Abdulrouf ${ }^{\prime}$ \\ Binny Thomas ${ }^{4,5}$ \\ Afif Ahmed' \\ Moza AlHail' \\ 'Department of Pharmacy, Women's \\ Hospital, Hamad Medical Corporation, \\ Doha, Qatar; ${ }^{2}$ Public Health Program, \\ London School of Hygiene and \\ Tropical Medicine, University \\ of London, UK; ${ }^{3}$ Department of \\ Obstetrics and Gynecology, Women's \\ Hospital, ${ }^{4}$ Clinical Support Service \\ Unit, Hamad Medical Corporation, \\ Doha, Qatar; ${ }^{5}$ Pharmacy and \\ Life Sciences Research Institute, \\ Robert Gordon University, \\ Aberdeen, Scotland
}

Background: Misoprostol is an effective medical method for the management of pregnancy loss. However, data on its efficacy and safety in women with previous cesarean deliveries are limited.

Case presentation: We report a 36-year-old patient, gravida 11 para 6, with a diagnosis of missed miscarriage at 15 weeks of gestation. The patient had a significant obstetric history of previous five cesarean deliveries and uterine rupture. Following patient counseling about the medical and surgical options of managing her miscarriage, the patient opted for medical method. Low-dose misoprostol of $100 \mu \mathrm{g}$ was inserted vaginally and repeated again after 6 hours. The patient had an uneventful complete miscarriage following the second dose of misoprostol. No uterine rupture, no extra vaginal bleeding, and no blood transfusion were observed.

Conclusion: We conclude that adopting a low-dose misoprostol protocol could be potentially safe and effective in managing second trimester missed miscarriage in women with repeated cesarean deliveries and/or uterine rupture history. Further studies are needed to confirm these results.

Keywords: cesarean section, uterine rupture, prostaglandin E1, misoprostol, second trimester, miscarriage, abortion

\section{Introduction}

Uterine rupture is one of the most catastrophic obstetric events that is associated with a high incidence of morbidity and mortality. Complications of uterine rupture can affect not only the current pregnancy but also future fertility and future pregnancy outcomes. Uterine rupture is more common in grand multiparous women and in those with previous cesarean sections. ${ }^{1,2}$

The rate of cesarean section is increasing worldwide. Consequently, the rate of women with a previous cesarean section is increasing and the risk of uterine rupture is becoming higher. Although the incidence rate of uterine rupture in women with previously scarred uterus remains low $(<1 \%)$, it is still considered as a threat for obstetricians. $^{2}$

Misoprostol, a prostaglandin E1 (PGE1) analog, is widely used for the medical management of pregnancy loss. Misoprostol is inexpensive and stable at room temperature and can be given orally, vaginally, sublingually, buccally, or rectally. However, its use for managing miscarriage cases is still off-label. ${ }^{3}$

Different protocols are available for the medical management of first and second trimester miscarriages using misoprostol. ${ }^{2,4,5}$ However, a definite misoprostol protocol
Correspondence: Doua AISaad Department of Pharmacy, Women's Hospital, Hamad Medical Corporation, PO Box 3050, Doha, Qatar

Tel +97444393340

Fax +97444393867

Email alsaad.doua@gmail.com 
with good efficacy and safety for termination of pregnancy in women with more than one previous cesarean delivery is lacking. ${ }^{6,7}$ This case report presents our experience with the use of misoprostol for the management of second trimester miscarriage in a high-risk patient with previous five cesarean sections and uterine rupture.

\section{Case presentation}

A 36-year-old patient, gravida 11 para 6, with an ultrasound diagnosis of "missed miscarriage", presented to our hospital with lower abdominal pain. She was $18+$ gestational weeks by the last menstrual period (LMP) and 15+ gestational weeks by the ultrasound. Her previous obstetric history was significant for five lower segment cesarean sections (LSCS), four miscarriages, and one uterine rupture.

In her fifth pregnancy, she had a fetal demise at $\sim 24$ weeks of gestation, which was medically terminated. The patient was induced once; however, details of the medical induction were not available to review. The pregnancy termination was complicated by uterine rupture, which was managed by laparotomy, hysterotomy, and uterine rupture repair. Two of the five LSCS were done electively following the uterine rupture repair. The patient was healthy otherwise.

On physical examination, the patient was vitally stable, and the abdomen was soft with no tenderness on palpation. Pelvic transabdominal ultrasound was requested to rule out scar rupture. The myometrium-bladder interface was preserved, and scar thickness was $5 \mathrm{~mm}$, which was normal for a previous cesarean section. ${ }^{8,9}$ All laboratory findings were within the normal ranges.

Conservative, medical, and surgical management options and the associated risks and benefits were discussed with the patient. She opted for medical management. Accordingly, a low-dose misoprostol of $100 \mu \mathrm{g}$ was inserted vaginally in the posterior fornix, and 6 hours later, a repeat dose was given. Two hours after the second dose, the patient started to have pain and contractions. She was vitally stable. Her vaginal examination revealed an opened cervical os, and tissues were felt in the vagina; hence, the patient was shifted to labor and delivery suite for monitoring. After receiving adequate analgesia, a pelvic transabdominal ultrasound was done for scar thickness, which showed a thinning out of the myometrium at a scar site measuring $2 \mathrm{~mm}$. Two hours later, the fetus was aborted that weighed $60 \mathrm{~g}$, and 20 units of oxytocin infusion was started. One hour later, the placenta was delivered completely. The estimated blood loss was $180 \mathrm{~mL}$. The patient was vitally stable all through with no extra bleeding.
The following day, the patient was stable with minimal vaginal bleeding and the repeated complete blood count (CBC) was normal; a prophylactic antibiotic amoxicillinclavulanate $625 \mathrm{mg}$ every 8 hours orally for 7 days was started. On Day 2 post miscarriage, the patient was discharged home with a follow-up appointment.

\section{Discussion}

To our knowledge, there are no published reports describing the outcomes of low-dose misoprostol in managing the second trimester missed miscarriage in women with previous five cesarean deliveries and a history of uterine rupture. Different protocols are available for the medical management of second trimester miscarriage in women with intact uterus. ${ }^{4,5}$ However, management options for cases with scarred uterus are limited and the potential risk for maternal complications is high. ${ }^{6,7}$ Hence, it is essential to report outcomes of management.

The optimal dose of misoprostol varies widely from 20 to $600 \mu \mathrm{g}$ depending on the indication and the gestational age. In women with a scarred uterus, lower doses of misoprostol are recommended due to the risk of uterine rupture. ${ }^{5}$ In this case report, a low-dose misoprostol of $100 \mu \mathrm{g}$ every 6 hours was considered to minimize the risk of uterine rupture while maintaining effectiveness.

Two systematic reviews published in 2009 evaluated the risk of uterine rupture in women with previous cesarean delivery who used misoprostol for the management of second trimester abortion. ${ }^{10,11}$ The risk of uterine rupture for women with or without previous cesarean delivery was similar. Nevertheless, there was insufficient information about uterine rupture risk in women with more than one previous cesarean delivery or with previous classical cesarean section, and information about optimum dosing regimen of misoprostol was missing. Accordingly, findings should be applied with caution.

A more recent systematic review and meta-analysis assessed the efficacy and safety of cervical ripening agents in the second trimester of pregnancy in patients with a previous cesarean delivery. ${ }^{12}$ The review concluded that PGE1 use was efficacious and was not associated with a significantly increased risk for uterine rupture among women with only one cesarean delivery. However, in women with two or more cesarean deliveries, the risk for uterine rupture increased significantly compared to that in women without a previous cesarean delivery, although the absolute risk appeared to be relatively small. The review results were limited by the variation in hospital protocols, doses, routes 
of administration, and nonuniform reporting of the number of previous cesarean deliveries ( 1 or $>1)$, as well as the lack of information regarding the types of previous uterine scar. The use of misoprostol in two women with previous three cesarean deliveries was described by Alsibiani. ${ }^{13}$ One woman received $800 \mu \mathrm{g}$ of oral misoprostol for the second trimester termination of pregnancy, while the other woman received $800 \mu \mathrm{g}$ of vaginal misoprostol for the first trimester missed miscarriage. Favorable results were reported concerning a single high dose of misoprostol use as part of abortion management, but the safety could not be ascertained from that study.

Overall, limited data are available regarding misoprostol safety in women with previous multiple cesarean deliveries, and available evidences are mainly dependent on individual practice and/or underpowered retrospective studies. Furthermore, there are inconsistencies about the optimum regimen of misoprostol, and the optimum dose is yet to be established.

\section{Conclusion}

Favorable results were obtained with low-dose misoprostol for the second trimester missed miscarriage management in a woman with previous repeated cesarean deliveries and a history of uterine rupture. Safety concerns such as uterine rupture and excessive bleeding were not encountered. However, the effectiveness and safety cannot be ascertained based on this case report solely.

\section{Acknowledgments}

This work was supported by the Hamad Medical Research Center, Hamad Medical Corporation, Qatar. Waiver of informed consent was obtained from the Hamad Medical Research Center (reference number: 16112/16).

\section{Disclosure}

The authors report no conflicts of interest in this work.

\section{References}

1. Murphy D. Uterine rupture. Curr Opin Obstet Gynecol. 2006;18: 135-140.

2. Manoharan M, Wuntakal R, Erskine K. Uterine rupture: a revisit. Obstet Gynaecol. 2010;12:223-230.

3. American College of Obstetricians and Gynecologists. Practice bulletin no. 143: medical management of first-trimester abortion. Obstet Gynecol. 2014;123(3):676-692.

4. World Health Organization [database on the Internet]. Safe Abortion: Technical and Policy Guidance for Health Systems. 2nd ed. Geneva: World Health Organization; 2012. Available from: https://www.ncbi nlm.nih.gov/books/NBK138196/. Accessed February 8, 2017.

5. Elati A, Weeks AD. The use of misoprostol in obstetrics and gynaecology. BJOG. 2009;116(suppl 1):61-69.

6. Dodd JM, Crowther CA. Misoprostol for induction of labour to terminate pregnancy in the second or third trimester for women with a fetal anomaly or after intrauterine fetal death. Cochrane Database Syst Rev. 2010;4:CD004901.

7. Mathews JE. Misoprostol for Induction of Labour to Terminate Pregnancy in the Second or Third Trimester for Women with a Fetal Anomaly or after Intrauterine Fetal Death: RHL Commentary (Last Revised: October 1, 2010); 2010. The WHO Reproductive Health Library; Geneva: World Health Organization.

8. Naji O, Abdallah Y, Bij De Vaate AJ, et al. Standardized approach for imaging and measuring cesarean section scars using ultrasonography. Ultrasound Obstet Gynecol. 2012;39(3):252-259.

9. Mohammed AB, Al-Moghazi DA, Hamdy MT, Mohammed EM Ultrasonographic evaluation of lower uterine segment thickness in pregnant women with previous cesarean section. Middle East Fertil Soc J. 2010;15(3):188-193.

10. Berghella V, Airoldi J, O’Neill AM, Einhorn K, Hoffman M. Misoprostol for second trimester pregnancy termination in women with prior caesarean: a systematic review. BJOG. 2009;116(9):1151-1157.

11. Goyal V. Uterine rupture in second-trimester misoprostol-induced abortion after cesarean delivery: a systematic review. Obstet Gynecol. 2009;113(5):1117-1123.

12. Andrikopoulou M, Lavery JA, Ananth CV, Vintzileos AM. Cervical ripening agents in the second trimester of pregnancy in women with a scarred uterus: a systematic review and meta-analysis of observational studies. Am J Obstet Gynecol. 2016;215(2):177-194.

13. Alsibiani SA. Misoprostol for pregnancy termination in grand multiparous women with three cesarean deliveries. Int $J$ Gynecol Obstet. 2009;106(3):255-256
Therapeutics and Clinical Risk Management

\section{Publish your work in this journal}

Therapeutics and Clinical Risk Management is an international, peerreviewed journal of clinical therapeutics and risk management, focusing on concise rapid reporting of clinical studies in all therapeutic areas, outcomes, safety, and programs for the effective, safe, and sustained use of medicines. This journal is indexed on PubMed Central, CAS,

\section{Dovepress}

EMBase, Scopus and the Elsevier Bibliographic databases. The manuscript management system is completely online and includes a very quick and fair peer-review system, which is all easy to use. Visit http://www.dovepress.com/testimonials.php to read real quotes from published authors. 